\title{
Laser-induced photoacoustic tomography enhanced with an optical contrast agent
}

Xueding Wang, Geng Ku, Xueyi Xie, Malgorzata A. Wegiel, Darryl J. Bornhop, et al.

Xueding Wang, Geng Ku, Xueyi Xie, Malgorzata A. Wegiel, Darryl J. Bornhop, George Stoica, Lihong V. Wang, "Laser-induced photoacoustic tomography enhanced with an optical contrast agent," Proc. SPIE 5320, Photons Plus Ultrasound: Imaging and Sensing, (12 July 2004); doi: $10.1117 / 12.532434$

SPIE. Event: Biomedical Optics 2004, 2004, San Jose, CA, United States 


\title{
Laser-induced photoacoustic tomography enhanced with an optical contrast agent
}

\author{
Xueding Wang ${ }^{\mathrm{a}}$, Geng Ku ${ }^{\mathrm{a}}$, Xueyi Xie ${ }^{\mathrm{a}}$, Malgorzata A. Wegiel ${ }^{\mathrm{b}}$, Darryl J. Bornhop ${ }^{\mathrm{c}}$, George Stoica ${ }^{\mathrm{d}}$, \\ and Lihong V. Wang ${ }^{* a}$ \\ ${ }^{a}$ Optical Imaging Laboratory, Department of Biomedical Engineering, Texas A\&M University, 3120 \\ TAMU, College Station, Texas 77843-3120 \\ ${ }^{\mathrm{b}}$ Department of Chemistry and Biochemistry, Texas Tech University, Lubbock, TX 79409-1061 \\ ${ }^{c}$ Department of Chemistry, Vanderbilt University, Nashville, TN 37235 \\ ${ }^{\mathrm{d}}$ Department of Pathobiology, Texas A\&M University, College Station, TX 77843-5547
}

\begin{abstract}
Optical contrast agents, such as indocyanine dyes, nano-particles and their functional derivatives, have been widely applied to enhance the sensitivity and specificity of optical imaging. However, due to the overwhelming scattering of light in biological tissues, the spatial resolution of traditional optical imaging degrades drastically as the imaging depth increases. For the first time to our knowledge, non-invasive in vivo photoacoustic imaging of an optical contrast agent, distributed in the rat brain, was implemented with near-infrared light. Injection of indocyanine green polyethylene glycol, a contrast agent with a high absorption at the 805-nm wavelength, into the circulatory system of a rat enhanced the absorption contrast between the blood vessels and the background brain tissues. Because near-infrared light can penetrate deep into the brain tissues through the skin and skull, we were able to successfully reconstruct the vascular distribution in the rat brain from the detected photoacoustic signals. The dynamic concentration of this contrast agent in the brain blood after the intravenous injection was also studied. This work proved that the distribution of an exogenous contrast agent in biological tissues can be imaged clearly and accurately by photoacoustic tomography. This new technology has high potential for application in dynamic and molecular medical imaging.
\end{abstract}

Keywords: photoacoustic tomography, contrast agent, indocyanine green, near-infrared light, neuroimaging

\section{INTRODUCTION}

The high sensitivity and specificity of optical imaging modalities aided by optical contrast agents parallels that of nuclear imaging and enables the visualization of distant organs, such as the brain, non-invasively. Indocyanine dyes, nano-particles and their functional derivatives have strong absorption in the near-infrared (NIR) spectra. NIR light is highly preferred in optical imaging because it has a comparatively lower absorption coefficient in biological tissues and, consequently, has a much deeper penetration depth compared to visible light. For this reason, optical contrast agents with absorption peaks in the NIR region are especially useful. For example, indocyanine green (ICG), in combination with NIR techniques, is employed widely in clinical applications such as cardiac output monitoring, ${ }^{1}$ hepatic function studies, ${ }^{2}$ angiography in ophthalmology, ${ }^{3,4}$ and tumor detection. ${ }^{5,6}$ However, due to the strongly scattering nature of biological tissues in NIR, the spatial resolution of pure optical imaging degrades significantly as the imaging depth increases. Therefore, traditional optical imaging techniques, whether diffuse optical tomography, fluorescence imaging, or other microscopies, cannot provide high resolution images of vasculature in biological tissues beyond the skin.

In comparison with light waves, acoustic waves are much less scattering in biological tissues and can directly furnish localization information for imaging. For this reason, ultrasonic signals can provide much better spatial resolution for the imaging of biological tissues beneath the skin. Photoacoustic tomography, ${ }^{7-13}$ as a hybrid imaging modality, combines the high contrast advantage of optical imaging with the high resolution advantage of ultrasonic imaging. After

*Corresponding author: email: Lwang@ tamu.edu; phone: 1979 847-9040; fax: 1979 845-4450 
previously achieving success in realizing non-invasive photoacoustic tomography of small-animal brains in vivo based on intrinsic optical contrast, ${ }^{12,13}$ in this work we study in vivo photoacoustic imaging of an exogenous contrast agent distributed in small-animal brains. The distributions of NIR absorption, enhanced by an ICG-based contrast agent in rat brain blood, were imaged with the photoacoustic modality. With this technique, we successfully achieved non-invasive photoacoustic angiography - mapping of the vasculature-in the rat brain.

\section{EXPERIMENTAL SETUP}

The experimental setup for non-invasive photoacoustic angiography in the rat brain with NIR light and an optical contrast agent is shown in Fig. 1. A tunable dye laser (ND6000, Continuum) pumped by an Nd:YAG laser (Brilliant B, Bigsky) was employed as the irradiation source. The FWHM of the laser pulse duration was $6.5 \mathrm{~ns}$; the pulse repetition rate was $10 \mathrm{~Hz}$. The laser beam was expanded and homogenized by a concave lens and a piece of ground glass before it reached the rat head. The incident energy density of the laser beam was controlled to $<2 \mathrm{~mJ} / \mathrm{cm}^{2}$ on the surface of the rat head, which induced a temperature rise in the brain vessels estimated to be $<3 \mathrm{mK}$. This incident energy density was much less than the maximum energy density (MPE) of the ANSI standard. ${ }^{14}$ A non-focused ultrasonic transducer (XMS310 , Panametrics) with a central frequency of $10.4 \mathrm{MHz}$, a bandwidth of $\sim 100 \%$ at $-6 \mathrm{~dB}$ and an active element of 2 $\mathrm{mm}$ in diameter was used to detect the photoacoustic signals. The transducer was driven by a computer-controlled step motor to scan around the rat brain cortical surface in the $x-y$ plane with a radius of $3 \mathrm{~cm}$ and a step size of $1.5 \mathrm{deg}$. For a circular scan, more than 200 steps were needed. The data acquisition time for one image was about 25 minutes. The photoacoustic signals detected by the ultrasonic transducer were sent to an oscilloscope (TDS 540B, Tektronics) through an amplifier. Finally, the digitized photoacoustic signals were collected by the computer for image reconstruction. Employing the full-view circular-scan geometry, the point-to-point absorption distribution in the rat brain was accurately reconstructed from the detected photoacoustic signals through a modified back-projection algorithm. ${ }^{15}$

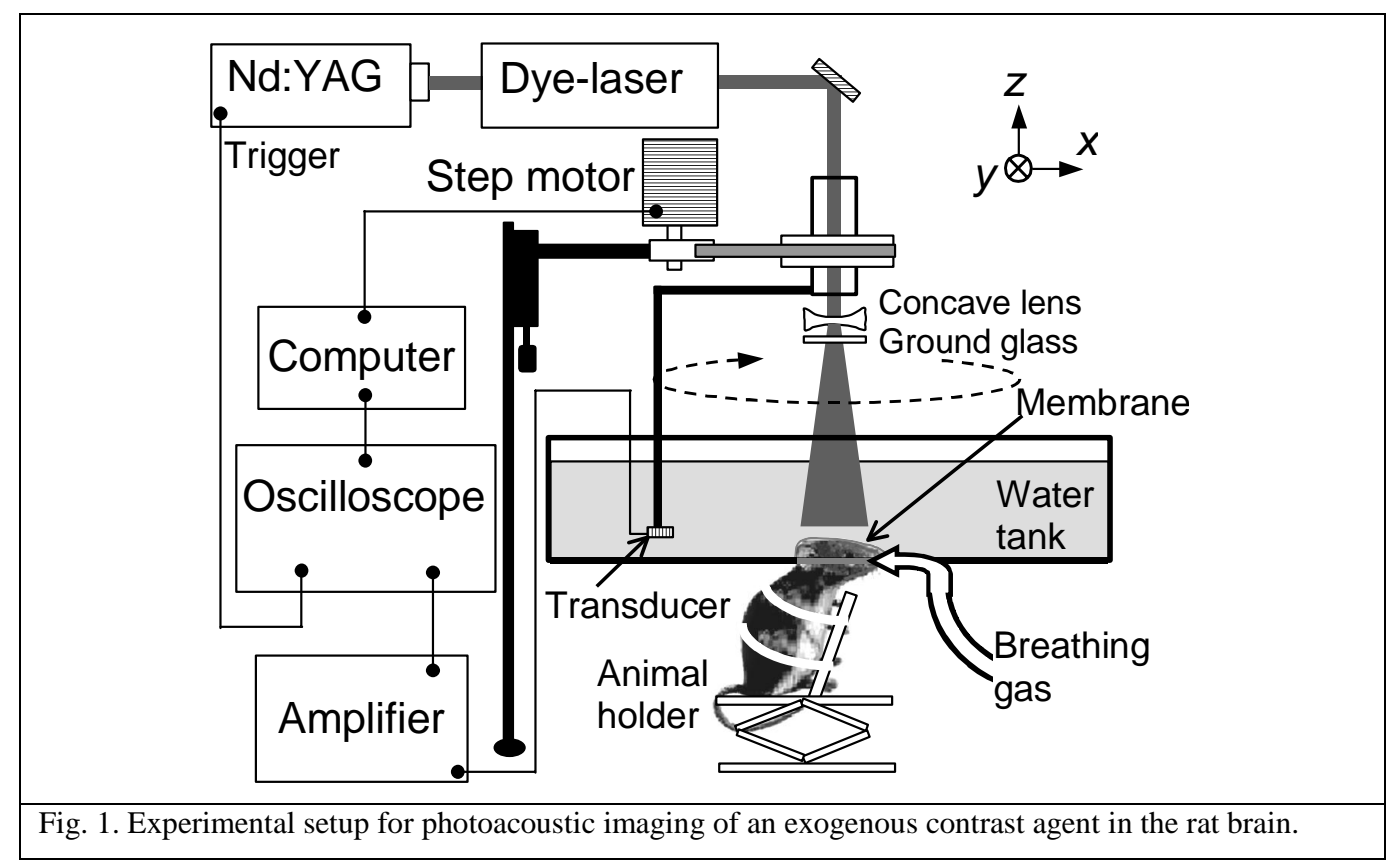

The rat was fixed by a homemade animal holder with its head protruding into the water tank through a hole in the bottom of the tank. A piece of clear membrane between the water and the rat head sealed the hole. A thin layer of ultrasonic coupling gel was applied on the surface of the rat head. Through a breathing mask, the rat could breathe freely during the in vivo experiment. Sprague Dawley rats ( $150 \mathrm{~g}$, Charles River Breeding Laboratories) were employed in 
this work. Before imaging, the hair on each rat's head was removed using hair remover lotion. A dose of $87 \mathrm{mg} / \mathrm{kg}$ Ketamine plus Xylasine $13 \mathrm{mg} / \mathrm{kg}$ administered intramuscularly was used to anesthetize the rat during the experiment. The optical contrast agent was injected into the circulatory system of the rat via the tail vein.

\section{OPTICAL CONTRAST AGENT}

With our current imaging system, the detection of the photoacoustic signals is realized through a circular-scan of a single-element transducer. Therefore, the data acquisition is slow. The image obtained by this setup represents a distribution of the averaged optical absorption during the period of data acquisition. In this case, an applied contrast agent with a prolonged clearance time benefits the acquisition of imaging signals, especially when several images need to be acquired after the intravenous injection. When an ultrasonic transducer array, instead of a single-element transducer, is adopted in the future, this technology will provide real-time and more accurate quantitative monitoring of a contrast agent distributed in biological tissues.

In order to prolong the clearance time of ICG in blood, polyethylene glycol (PEG), ${ }^{16}$ an FDA approved polymer, was adopted to stabilize ICG. This ICG-based contrast agent (ICG-PEG) has a high absorption from the 750-nm to the 850nm wavelength (see Fig. 2). Considering the pumping efficiency of our dye laser, we used laser pulses at the 805-nm wavelength in this work. The ICG-PEG in the PBS buffer was reconstituted with saline $\left(\mathrm{pH}=7.4\right.$; concentration=3.2 $\times 10^{-}$ ${ }^{4} \mathrm{M}$ ) and then injected intravenously at a dosage of $0.25 \mathrm{ml} / 100 \mathrm{~g}$ body weight, which led to an estimated ICG concentration of $\sim 1 \times 10^{-5} \mathrm{M}$ in the rat blood. With this dosage and the applied laser energy density for this experiment, the magnitude of the photoacoustic signals received by the ultrasonic transducer was on the order of $10 \mu \mathrm{V}$.

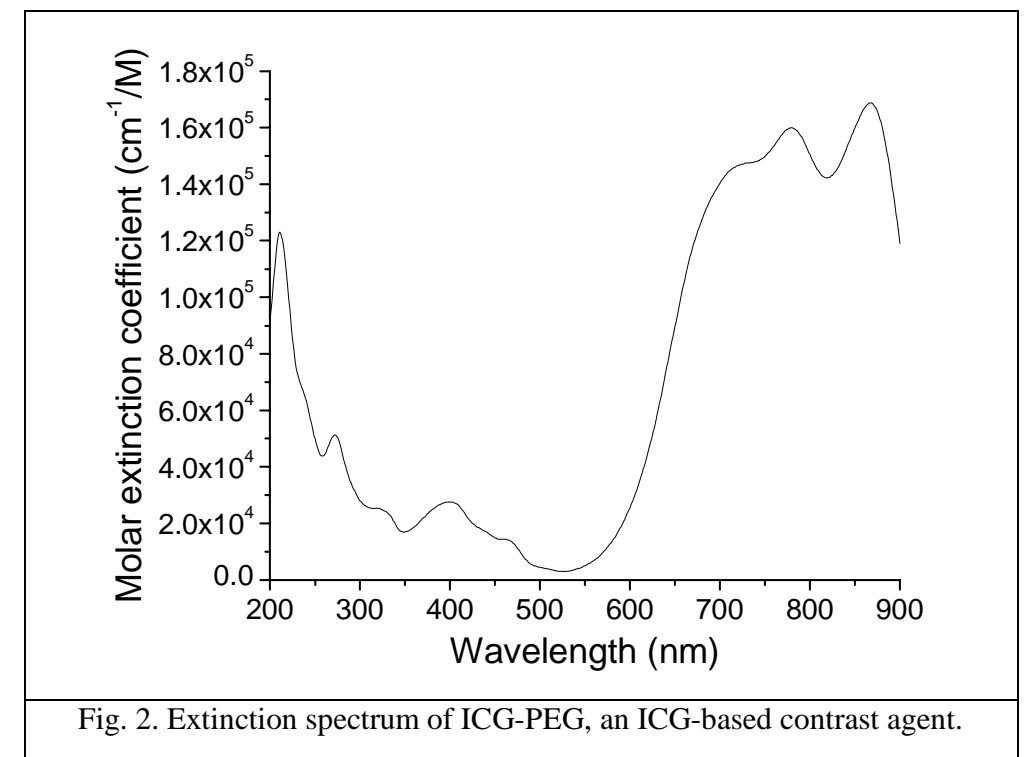

\section{RESULTS}

\subsection{In situ imaging of the rat brain}

Photoacoustic angiographs of the rat brain in situ based on intrinsic and extrinsic optical contrasts were compared to verify the improvement in imaging quality brought about by the contrast agent (see Fig. 3). The brain image in Fig. 3(A) was obtained from a sacrificed rat without contrast enhancement. The brain image in Fig. 3(B) was obtained from a rat sacrificed 5 minutes after the intravenous injection of ICG-PEG. The two brain images use the same gray scale. 
Figs. 3(C) and (D) are open-skull photographs of the cortical vascular distributions in rat brains corresponding to the images in Figs. 3(A) and (B), respectively. With the contrast enhancement brought about by the ICG-PEG, the image in Fig. 3(B) maps the brain vessels clearly with satisfactory spatial resolution and matches well with the open-skull photograph of the anatomy obtained after the imaging experiment.

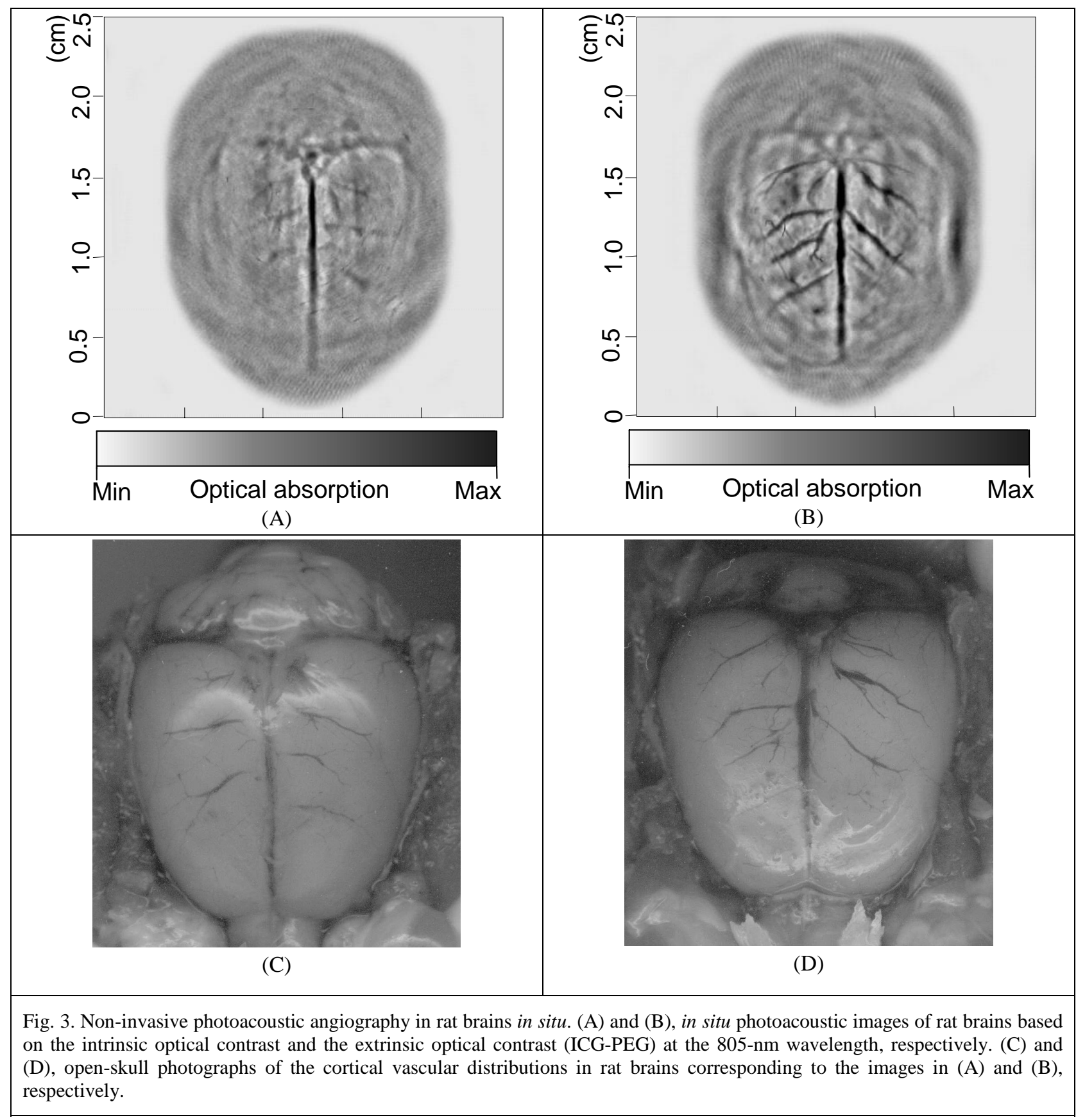

\subsection{In vivo imaging of the rat brain}


Non-invasive photoacoustic angiography in rat brains in vivo with NIR light and ICG-PEG contrast agent was realized (see Fig. 4). Both of the two brain images in Figs. 4(A) and (B) were obtained after the intravenous injection of the contrast agent while the image in Fig. 4(A) was acquired 0 to 25 minutes after the injection and the image in Fig. 4(B) was acquired 60 to 85 minutes after the injection. By comparing these two brain images, we can see that the image quality, in other words, the contrast between the blood vessels and the background brain tissues, degraded as a result of the clearance of ICG-PEG from the blood.

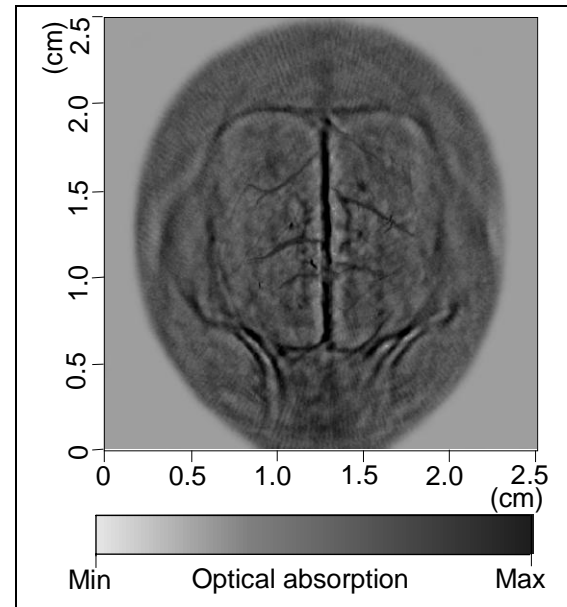

(A)

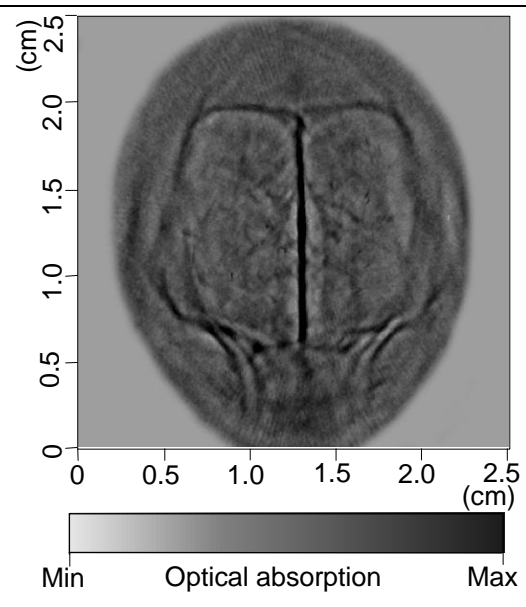

(B)

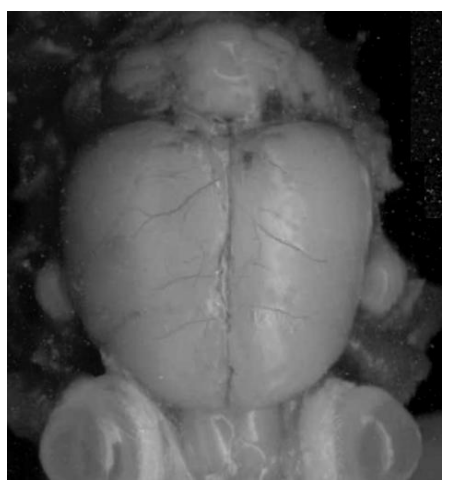

(C)

Fig. 4. Non-invasive photoacoustic angiography in a rat brain in vivo with NIR light and ICG-PEG contrast agent. (A) The brain image acquired 0 to 25 minutes after the intravenous injection of ICG-PEG. (B) The brain image acquired 60 to 85 minutes after the injection. (C) The open-skull photograph of the anatomy obtained after the imaging experiment.

\section{CONCLUSION}

An ICG-based optical contrast agent in the circulatory system enhances the absorption contrast between the blood vessels and the background tissues in the rat brain. Based on photoacoustic imaging of the exogenous contrast agent with NIR light, non-invasive angiography in rat brains that yielded high contrast and high spatial resolution was implemented both in situ and in vivo. This new technology has potentially wide application in diagnostic imaging and therapeutic monitoring. Because this technique provides an accurate non-invasive method for monitoring fluid pathways in biological tissues, it can be employed in imaging vascular changes in tumors, delineating neovascularization, and studying global and regional hemodynamic activities in the brain. Since the contrast agent is expected to accumulate in neoplastic tissues or traumatized regions, this technique is also promising for determining the margins of embedded tumors or bruises with a high degree of accuracy. More importantly, since contrast agents can be conjugated to bioactive materials, such as peptides, proteins, antibodies, hormones, and drugs, this technique can be readily extended to functional imaging at molecular and genetic levels.

\section{REFERENCES}

1. Y.-L. He, H. Tanigami, H. Ueyama, T. Mashimo, and I. Yoshiya, "Measurement of blood volume using indocyanine green measured with pulse-spectrometry: Its reproducibility and reliability," Crit. Care Med. 26, 14461451 (1998).

2. J. Caesar, S. Shaldon, L. Chiandussi, L. Guevara, and S. Sherlock, "The use of Indocyanine green in the measurement of hepatic blood flow and as a test of hepatic function," Clin. Sci. 21, $43-57$ (1961).

3. B. F. Hochheim, "Angiography of retina with indocyanine green," Arch. Ophthalmol. 86, 564-565 (1971). 
4. L. A. Yannuzzi, J. S. Slakter, J. A. Sorenson, D. R. Guyer, and D. A. Orlock, "Digital indocyanine green videoangiography and choroidal neovascularization," Retina-J. Ret. Vit. Dis. 12, 191-223 (1992).

5. M. M. Haglund, M. S. Berger, and D. W. Hochman, "Enhanced optical imaging of human gliomas and tumor margins," Neurosurgery 38, 308-317 (1996).

6. V. Ntziachristos, A. G. Yodh, M. Schnall, and B. Chance, "Concurrent MRI and diffuse optical tomography of breast following indocyanine green enhancement," Proc. Natl. Acad. Sci. USA 97, 2767-2772 (2000).

7. C. G. A. Hoelen, F. F. M. de Mul, R. Pongers, and A. Dekker, Three-dimensional photoacoustic imaging of blood vessels in tissue. Opt. Lett. 23, 648-650 (1998).

8. R. A. Kruger, D. R. Reinecke, and G. A. Kruger, Thermoacoustic computed tomography-technical considerations. Med. Phys. 26, 1832-1837 (1999).

9. R. O. Esenaliev, A. A. Karabutov, and A. A. Oraevsky, Sensitivity of laser opto-acoustic imaging in detection of small deeply embedded tumors. IEEE J. Sel. Top. Quant. 5, 981-988 (1999).

10. A. A. Karabutov, E. V. Savateeva, and N. B. Podymova, "Backward mode detection of laser-induced wide-band ultrasonic transients with optoacoustic transducer,”J. Appl. Phys. 87, 2003-2014 (2000).

11. K. P. Köstli, D. Frauchiger, J. J. Niederhauser, G. Paltauf, H. P. Weber, and M. Frenz, Optoacoustic imaging using a three-dimensional reconstruction algorithm. IEEE J. Sel. Top. Quant. 7, 918-923 (2001).

12. X. Wang, Y. Pang, G. Ku, X. Xie, G. Stoica, and L. V. Wang, "Non-invasive laser-induced photoacoustic tomography for structural and functional imaging of the brain in vivo," Nat. Biotech. 21, $803-806$ (2003).

13. X. Wang, Y. Pang, G. Ku, G. Stoica, and L. V. Wang, "Three-dimensional laser-induced photoacoustic tomography of the mouse brain with the skin and skull intact," Opt. Lett. 28, 1739-1741 (2003).

14. American National Standards Institute, American national standard for the safe use of lasers. Standard Z136.1-2000. (ANSI, Inc., New York, NY, 2000).

15. M. Xu and L. V. Wang, "Time-domain reconstruction for thermoacoustic tomography in a spherical geometry," IEEE T. Med. Imag. 21, $814-822$ (2002).

16. S. Zalipsky, E. Brandeis, M. S. Newman, and M. C. Woodle, "Long circulating, cationic liposomes containing amino-PEG-phosphatidylethanolamine," FEBS Lett. 353, 71-74 (1994). 